\title{
Thymol-Induced Chemical Leukoderma Successfully Treated with 308-Nanometer Excimer Laser
}

\author{
Maximillian A. Weigelt Alexander T. Herbst Antonella Tosti Hadar Lev-Tov \\ Dr. Phillip Frost Department of Dermatology and Cutaneous Surgery, University of Miami - Miller School of \\ Medicine, Miami, FL, USA
}

\section{Established Facts}

- Chemical leukoderma is an acquired depigmenting disorder caused by repeated exposure to chemical compounds.

- A thorough history and physical exam are instrumental to rule out other causes of depigmentation, such as vitiligo and post-inflammatory leukoderma.

\section{Novel Insights}

- Here, we present the second reported case of chemical leukoderma induced by thymol.

- Partial re-pigmentation was achieved after 32 treatments with 308-nm excimer laser.

- Physicians should be cognizant of the largely unregulated components of cosmetic products.

\section{Keywords}

Contact · Chemical · Leukoderma · Laser · Depigmentation

\begin{abstract}
Introduction: Chemical leukoderma (CL) is an acquired depigmenting disorder caused by repeated exposure to chemical compounds. Thymol is a phenol derivative used as a preservative or antiseptic in many commercially available products. Here, we present the second reported case of CL caused by a thymol-containing compound. Case Presentation: A 48-year-old woman presented with a 4-month history of depigmentation of the nail folds of all ten fingers. This occurred after 1 month of twice-daily application of a thymol-contain-
\end{abstract}

ing compound intended for the removal of gel nails. No improvement was noted after the product was discontinued. There was no family history of vitiligo or other autoimmune disorders. On physical exam, depigmentation of all ten proximal and lateral nail folds was seen, with accentuation on Wood's lamp exam. Partial re-pigmentation was achieved after 32 treatments with 308-nm excimer laser. Discussion: A thorough history and physical exam are instrumental in differentiating $C L$ from other causes of depigmentation. Avoidance of the offending agent is an essential part of management. It is important to note that many cosmetic products are not tightly regulated by the FDA. Excimer laser is an effective treatment for $C L$ with a favorable side-effect profile.

(c) 2020 S. Karger AG, Basel karger@karger.com www.karger.com/sad

(C) 2020 S. Karger AG, Basel

Karger ${ }^{\prime}=$
Maximillian A. Weigelt

Dr. Phillip Frost Department of Dermatology and Cutaneous Surgery University of Miami - Miller School of Medicine

1321 NW 14th Street, West Building, Suite 505, Miami, FL 33125 (USA) mxw868@miami.edu 


\section{Introduction}

Chemical leukoderma (CL, also known as contact leukoderma, contact vitiligo, or contact depigmentation) is an acquired depigmenting disorder caused by repeated exposure to chemical compounds. Phenols and catecholamines are the most common and best-studied group of offending agents [1]. Thymol is a phenol derivative used as a preservative or antiseptic in many commercially available medications and cosmetics [2]. Here, we present, to our knowledge, the second reported case of CL caused by thymol or thymol-containing compounds and successful treatment with 308-nm excimer laser.

\section{Case Presentation}

A 48-year-old black woman presented to the clinic with a 4-month history of depigmentation of the nail folds of all her fingernails. She had previously developed nail roughness after removal of gel nails for which she began applying "Thymolize," a product containing thymol and denatured alcohol, recommended by her manicurist. After 1 month of twice-daily application, the patient developed progressive asymptomatic white depigmentation around each nail fold. She then discontinued the Thymolize product, but no spontaneous improvement was noted. There was no family history of vitiligo, thyroid disease, or other autoimmune disorder. Past medical history was unremarkable.

Physical exam was significant for depigmented patches affecting all ten proximal and lateral nail folds (Fig. 1) - the lesions accentuated on Wood's lamp exam. No nail dystrophy was noted. Her dermatological exam was otherwise normal. The patient was started on twice-weekly excimer laser treatments; after 3 months of treatment, marked improvement was noted in the left hand, while the right hand improved only slightly (Fig. 2).

\section{Discussion}

CL affects $0.5-1 \%$ of the population and is often mistaken for other forms of acquired depigmentation such as vitiligo [1]. It characteristically presents with numerous small pea-sized or "confetti" depigmented macules which progressively coalesce. Distal lesions are found in over $25 \%$ of patients, often contributing to a misdiagnosis of acral vitiligo [3]. Symptoms are rare but itching can occur, especially if associated with contact dermatitis [1]. CL follows a progressive course, while vitiligo is cyclic and unpredictable. Repeated exposure to a chemical agent is necessary for the development of CL; in the case of depigmentation occurring after a single exposure, post-inflammatory leukoderma and vitiligo-associated Koebnerization should be excluded [3].

Thymol-Induced Chemical Leukoderma

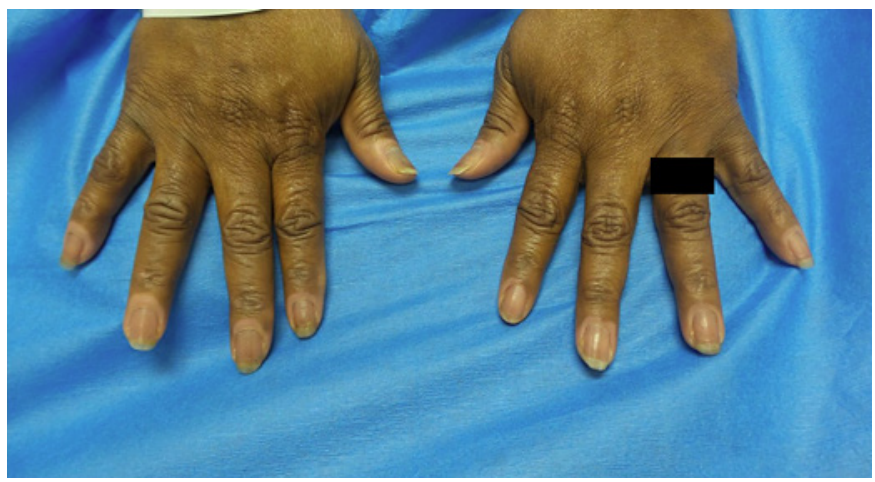

Fig. 1. Depigmentation of all ten nail folds after 1 month of twicedaily application of a thymol-containing product for removal of gel nails.

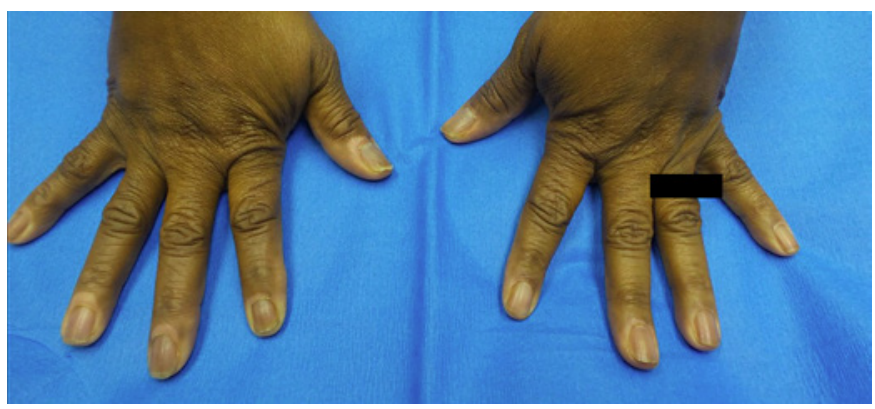

Fig. 2. Partial re-pigmentation of the nail folds after 32 biweekly treatments with 308-nm excimer laser, starting at $250 \mathrm{~mJ} / \mathrm{cm}^{2}$ and gradually increasing to $1,190 \mathrm{~mJ} / \mathrm{cm}^{2}$.

CL occurs only in certain susceptible individuals; the pathogenesis involves selective cytotoxicity of melanocytes, although the exact mechanism is currently unknown [1]. Phenols such as thymol are structurally similar to tyrosine and inhibit the melanin synthesis pathway via competitive inhibition of tyrosinase [1]. The cytotoxic effects of these compounds may be due to their conversion into free radicals and reactive oxygen species by tyrosinase-related protein 1 , which has also been found to be overexpressed in vitiligo [1]. Thymol has previously been reported as the cause of CL associated with Vick's VapoRub [2]. Interestingly, thymol esters have been found to exhibit significant depigmenting and cytotoxic activity in vitro, while thymol alone demonstrated none [4]. The Thymolize product used by this patient contained 3\% thymol, 5\% methanol, 9\% isopropanol, and $81 \%$ ethanol. It is important to remember that many over-the-counter preparations are not tightly regulated by the FDA. 
The 308-nm excimer is an effective treatment option for CL with a favorable side-effect profile $[5,6]$. The mechanism of action by which excimer causes re-pigmentation is thought to be due to endothelin-1-related migration and proliferation of melanocytes [7]. In a case of $p$-phenylenediamine- and hydrogen peroxide-induced leukoderma on the face, biweekly treatment starting at $200 \mathrm{~mJ} / \mathrm{cm}^{2}$ and gradually increasing to $1,400 \mathrm{~mJ} / \mathrm{cm}^{2}$ resulted in total re-pigmentation after 39 total treatments [6]. Another group used a similar protocol to treat amyl nitrate-induced CL, progressing from 100 to $320 \mathrm{~mJ} / \mathrm{cm}^{2}$, with total re-pigmentation occurring after 42 treatments $[5,6]$.

For our patient, we started with $250 \mathrm{~mJ} / \mathrm{cm}^{2}$ and gradually increased to $1,190 \mathrm{~mJ} / \mathrm{cm}^{2}$ for 32 total treatments per our institution's standardized dosing schedule which is based on skin type. After 32 treatments, the photo in Figure 2 was taken. The patient is pleased with the results and treatment is ongoing. The observed difference in treatment response between the two hands could possibly be explained by variation in the amount of product applied at the initial exposure, that is, more product may have been applied to the right hand by the less dexterous left.

In summary, $\mathrm{CL}$ is a common acquired depigmenting disorder resulting from repeated exposure to a chemical agent. A thorough history is instrumental in differentiating between CL and other similar disorders, such as vitiligo, vitiligo-related Koebnerization, and post-inflammatory leukoderma. This is the second reported case of
CL caused by a thymol-containing compound. Physicians should be cognizant of the largely unregulated components that make up cosmetic products. In the presented case, excimer laser was effective in stimulating at least partial re-pigmentation. Nonetheless, avoidance of the offending agent remains central to management.

\section{Statement of Ethics}

This research was conducted ethically in accordance with the World Medical Association Declaration of Helsinki. The subject described in this case has given written informed consent to publish photos and the details of the case.

\section{Disclosure Statement}

The authors have no relevant disclosures.

\section{Funding Sources}

This work was not supported by any funding.

\section{Author Contributions}

All authors made substantial contributions to the conception and design of the work, were responsible for drafting the work and revising it critically, gave their final approval of the version to be published, and agreed to be accountable for questions related to the accuracy and integrity of the work.

\section{References}

1 Bonamonte $\mathrm{D}$, Vestita M, Romita P, Filoni A, Foti C, Angelini G. Chemical leukoderma. Dermatitis. 2016;27(3):90-9.

2 Boyse KE, Zirwas MJ. Chemical leukoderma associated with Vicks VapoRub. J Clin Aesthet Dermatol. 2008;1(4):34-5.

3 Saleem MD, Oussedik E, Schoch JJ, Berger AC, Picardo M. Acquired disorders with depigmentation: a systematic approach to vitiliginoid conditions. J Am Acad Dermatol. 2019;80(5):1215-31.e6.
4 Kang HH, Rho HS, Hwang JS, Oh SG. Depigmenting activity and low cytotoxicity of alkoxy benzoates or alkoxy cinnamte in cultured melanocytes. Chem Pharm Bull. 2003;51(9): 1085-8.

5 Vine K, Meulener M, Shieh S, Silverberg NB. Vitiliginous lesions induced by amyl nitrite exposure. Cutis. 2013;91(3):129-36.

6 Ghazi E, Ragi J, Milgraum S. Treatment of chemical leukoderma using a 308-nm excimer laser. Dermatol Surg. 2012;38(8):1407-9.

7 Beggs S, Short J, Rengifo-Pardo M, Ehrlich A. Applications of the excimer laser: a review. Dermatol Surg. 2015;41(11):1201-11. 